\title{
Phoneutrismo en Ecuador: reporte de un caso clínico
}

\author{
Susana Peñafiel [1], Andrea Tufiño [1], Diego Herrera [2] \\ 1 Postgrado de Medicina Familia PUCE Quito. Ecuador \\ 2 Saludesa Ecuador. Ecuador \\ PRÁCTICA FAMILIAR RURAL | Vol.2 | No.3 | Noviembre 2017 | Recibido: \\ 17/08/2017| Aprobado: 9/11/2017 \\ Como citar este artículo \\ Peñafiel S, Tufiño A, Herrera D. Phoneutrismo en Ecuador: reporte de un \\ caso clínico. Práctica Familiar Rural. 2018 noviembre; 2(3).
}

\section{RESUMEN}

Se presenta un caso de mordedura por Phoneutria nigriventer atendido en el Hospital Pedro Vicente Maldonado ubicado en el cantón del mismo nombre en la zona subtropical del Noroccidente de Pichincha, en el año 2017. El caso se trata de una paciente femenina de 40 años de edad la cual, mientras trabaja en tareas agrícolas sufre mordedura de araña bananera en dedo índice de mano izquierda, acudió a sala de emergencia presentando un cuadro de envenenamiento leve el cual evolucionó favorablemente. El phoneutrismo es un aracnidismo pocas veces reportado en Ecuador, debido posiblemente al sub registro de estos accidentes.

El objetivo del presente trabajo es fortalecer conocimientos y motivar a un adecuado registro, además, dar a conocer la importancia del manejo eficaz y oportuno debido a la ausencia de antídoto.

Palabras clave: aracnidismo, mordeduras de arañas, neurotóxicos

\section{Clinical Case: Phoneutrism in Ecuador}

\section{ABSTRACT}

A case of bite by Phoneutria nigriventer is presented treated at the Hospital Pedro Vicente Maldonado located in the canton of the same name in the subtropical zone of the Northwest of Pichincha, in the year 
2017. The case is about a female patient of 40 years of age which, while working in agricultural tasks, suffers banana's spider bite on left index finger, went to emergency room presenting a picture of mild poisoning which evolved favorably. Phonenutrism is an arachnidism rarely reported in Ecuador, possibly due to the sub-registry of these accidents.

The objective of this work is to strengthen knowledge and motivate an adequate record, in addition, to make known the importance of effective and timely management due to the absence of an antidote.

Key words: arachnidism, spider bites, neurotoxins

\section{Introducción}

El "phoneutrismo" o "ctenismo", es un aracneismo ocasionado por la mordedura e inoculación de veneno por el género Phoneutria spp, un arácnido de amplia distribución en Sudamérica y Centroamérica y sobretodo en regiones tropicales. De las ocho especies que se conocen hasta el momento, cuatro han sido reportadas en Ecuador, en la costa norte y la selva con climas tropicales propicios para su habitad, e incluyen a P.boliviensis, P.reidy, P.fera y P.nigriventer.

El aracneismos por Phoneutria nigriventer forman parte de las cuatro especies de arácnidos más venenosos según la OMS, por lo que es un importante problema de salud pública. No tenemos registro de la frecuencia de los casos, ni las formas clínicas en el Ecuador, se tienen reportes de casos en Colombia, Perú, y muchos más en Brasil.

El objetivo del siguiente reporte es motivar a reconocer de manera adecuada y oportuna el cuadro clínico de éste envenenamiento para poder brindar al paciente el manejo oportuno y eficaz del mismo, alertar sobre el sub registro de estos accidentes, debido, al desconocimiento del personal médico y paramédico. Se recalca la importancia y trascendencia de estos eventos para la Salud Pública, puesto que en Ecuador, no contamos con el antídoto.

\section{Presentación del caso}

Paciente mujer de 40 años, que mientras se encontraba realizando tareas agrícolas sufre mordedura de araña en dedo índice de mano izquierda, presentando posteriormente cuadro de malestar general y dolor importante en mano, se auto médica analgésico y medicina empírica sin mejoría por lo que acude una hora posterior al evento. La paciente identifico la araña y describe sus características.

Al examen físico se evidenciaba en dedo índice de mano izquierda edema y eritema hasta tercio medial de dorso y palma de la mano, se evidencia estigma de zona de mordedura con edema de dedo índice en relación a contralateral de $2 \mathrm{~cm}$, movimientos de flexión y extensión limitados.

Dentro de los posibles diagnósticos diferenciales incluyen mordeduras de diferentes artrópodos como hormigas, pulgas, chinches, garrapatas, ácaros, mosquitos y moscas mordedoras, así como erisipelas, celulitis, ectima, vasculitis, pioderma, zoster, urticaria, angioedema y quemaduras. Además de mordedura por ofidios.

Se realizan exámenes de laboratorio biometría más función renal y glicemia normales.

Se inicia terapia sintomática con bloqueadores H1, bloqueadores H2, hidratación analgesia y monitoreo de signos vitales, paciente es catalogada como mordedura de araña Phoneutria, con cuadro de envenenamiento leve evoluciona satisfactoriamente 

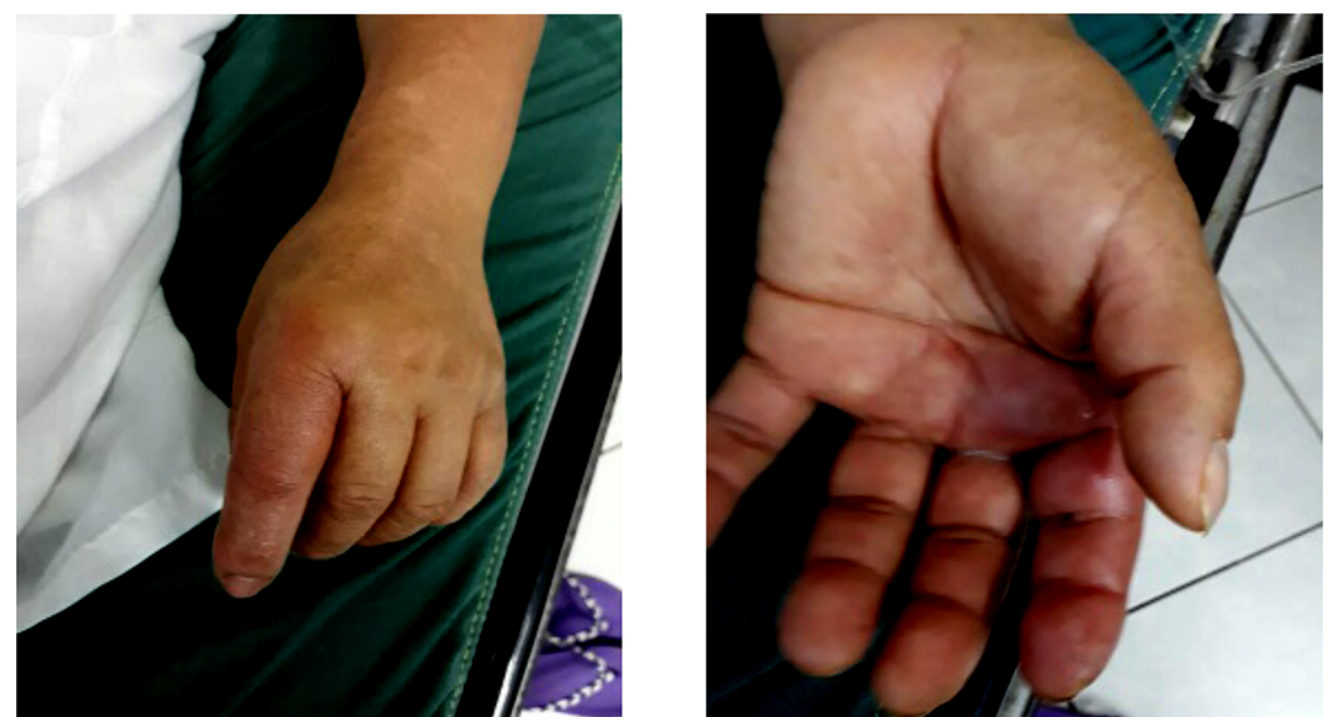

\section{Discusión}

El número de arañas conocidas a nivel mundial es de aproximadamente 1.7 millones, el orden de las arañas forma la clase de los arácnidos e integra el Phylum de los Artrópodos; representando sólo el $10 \%$ de todas las especies que habitan en el planeta, sin embargo sólo se han descrito de 34000 a 35000 especies, de las cuales se conoce que 12 de ellas pueden son potencialmente tóxicas para los humanos, y sólo 4 especies son de importancia médicas dada la casuística mundial de envenenamiento reportados. Éstas 4 especies son Loxosceles, Lactrudectus, Athrax y Phoneutria. [2,5].

Las arañas del género Phoneutria pertenecen a la familia de Ctenidae, se reportan mordeduras en toda América del Sur y Costa Rica. Sin embargo, la mayoría de los informes clínicamente importantes las mordeduras son de Brasil. La araña vagabunda sudamericana, Phoneutria nigriventer, es popularmente conocida como la "araña armada" o "araña platanera ", Phoneutria es la especie que más accidentes produce en seres humanos (1).

Phoneutria es una araña nocturna y solitaria, que no construyen redes, ellas captan presas mediante la caza activa y recorriendo grandes distancias por la noche. Ocasionalmente entran en las casas de las personas y cuando son descubiertas adquieren la posición agresiva característica.
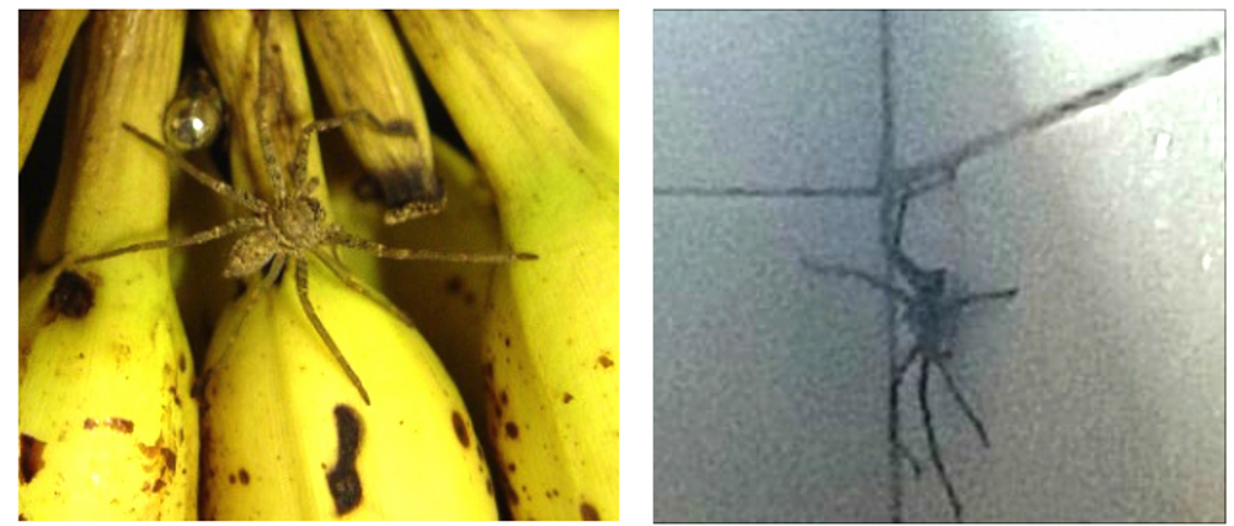
De acuerdo a su estudio taxonómico, son arañas de gran tamaño, con cefalotórax de hasta 4,5 cm; y una longitud total, incluida las patas, de hasta $15-16 \mathrm{~cm} \mathrm{[2,3].} \mathrm{Se} \mathrm{identifica} \mathrm{y} \mathrm{diferencia} \mathrm{de} \mathrm{otras}$ especies por la presencia de una densa escópula, asimismo por la característica presencia defensiva, adoptando una posición casi erecta y con movimiento lateral del cuerpo y piernas elevadas. Asimismo hay otras características que las diferencian como la coloración grisácea a castaño oscuro del cefalotórax; abdomen con dos filas de manchas dorsales más claras; piernas con salientes aguijones oscuros, con la base blanca; quelíceros con la cara frontal cubierta con vellos alargados y anaranjados que suelen tornarse rojos al encontrarse irritadas; y finalmente ocho ojos dispuestos en dos filas con fórmula 2-4-2[5,6].

Las Phoneutrias, son arañas errantes y muy agresivas, suelen estar en lugares oscuros y húmedos. A veces habitan cerca de las casas y tienen un gran poder defensivo poniéndose erectas y balanceándose hacia los costados antes de atacar. Son también llamadas arañas bananeras, habitan en selvas y suelen encontrarse en cajas de frutas. Son agresivas y construyen cuevas, superan los $4 \mathrm{~cm}$ de largo. Su veneno es neurotóxico actuando en los canales de sodio, la letalidad de las picaduras de arañas siempre está asociada al tiempo que hace que no comen es decir que no han aplicado el veneno. La muerte provocada por las Phoneutria Fera junto a la Phoneutria nigriventer puede ocurrir en horas especialmente en niños, en adulto generalmente solo produce edemas y dolores locales. En casos graves se agregan convulsiones, opistótonos, hipotensión, edema agudo de pulmón, shock y paro cardiorrespiratorio. (1)

La mayoría de accidentes ocurren en los primeros meses del año (marzo-mayo), ya que coinciden con su periodo de apareamiento, donde buscan el interior de los hogares y se alojan en lugares oscuros, como el interior de zapatos y en armarios. Las regiones anatómicas más afectadas suelen ser manos y pies, debido a que se relacionan con actividades de calzarse los zapatos, limpieza de jardines, manipulación de frutas o verduras, y otros [3,9]. Adicional al ciclo reproductivo hay otros factores que aumentan la incidencia de éstos accidentes como la temperatura y humedad [11].

\section{Cuadro clínico}

inmediato de gravedad variable que está asociada con la diaforesis, piloerección y eritema, a menudo sin evidencia de marcas de colmillos. El dolor se irradia proximalmente a la extremidad mordida. Al examen físico podemos encontrar taquicardia y ansiedad. En una serie de 422 mordeduras confirmadas casi el 90\% tenían envenenamiento leve (1)

Envenenamiento Moderado.- Al cuadro anterior se suman, síntomas no específicos como náuseas, vómitos, y mareos; y los efectos autonómicos tales como taquicardia, hipertensión, profusa diaforesis, salivación, trastornos visuales y priapismo (especialmente en los niños).

Envenenamiento grave.- Es más frecuente en niños menores de 10 años. Esta caracterizado por vómitos persistentes disnea, edema pulmonar, shock, y muerte en raras ocasiones, son menos del $1 \%$ de todas las mordeduras(1)

\section{Pautas de tratamiento}

1. Se recomienda la observación de los pacientes durante un lapso no menor a 6 horas desde la mordedura, para evaluar el eventual compromiso sistémico que puede ocasionar este envenenamiento, especialmente en los niños pequeños. (3)

2. Los casos moderados deberán internarse en sala general y los graves deberán ser trasladados, en lo posible, a unidades de terapia intensiva.

3. El tratamiento de los hallazgos locales agudos después de una mordedura de araña implica el cuidado local de la herida, el manejo del dolor, y, si está indicado, la profilaxis del tétanos.

4. Las medidas iniciales de tratamiento después de cualquier picadura de araña incluyen: 
- Limpie la mordida con jabón suave y agua.

- Mantenga la parte afectada del cuerpo en una posición elevada o neutral (si es posible).

- Administrar analgésicos según sea necesario, dependiendo de la respuesta del paciente a medicamentos antiinflamatorios no esteroideos, se puede requerir opioides.

- Administrar la profilaxis del tétanos si se indica.

5. La mayoría de las mordeduras se pueden manejar con una intervención mínima y sanar sin cicatrices. Las mordeduras deben ser monitoreadas para el desarrollo de la infección bacteriana secundaria con cobertura para estafilococo y estreptococo, como se recomienda para la celulitis sin complicaciones.

6. Los antibióticos se prescriben sólo si hay signos de infección como el aumento del eritema, fluctuación y supuración.

7. Tratamiento sintomático, puede incluir la infiltración de anestesia local del sitio de la mordedura.

8. Corticoides han sido utilizados, pero su eficacia no ha sido comprobada

9. El uso del antídoto está reservado para casos de envenamiento moderado o severo. (1)

El anti veneno, se ha utilizado para tratar el envenenamiento por mordeduras de araña de Phoneutria en Brasil desde 1925, y es un anti veneno polivalente $\mathrm{F}$ ( $\left.a b^{\prime}\right) 2$ usado para mordedura de araña, Phoneutria y Loxosceles y picadura de escorpión del género Tityus. La administración del antídoto en las primeras tres horas, está asociado con recuperación en $24 \mathrm{~h}$.

En resumen se requiere un alto índice de sospecha de picadura por araña, es necesario al no contar con las evidencias suficientes descartar otros eventos por reptiles como mordeduras por serpientes. Un diagnóstico presuntivo de una picadura de araña se basa más a menudo en la historia y la presentación clínica. Sin embargo, el diagnóstico de una picadura de araña se puede considerar definitiva solamente si se cumplen los criterios siguientes:

- Una araña fue observada infligiendo la mordedura.

- La araña fue recuperada, recogida y debidamente identificada.

- Existe una lesión cutánea y / o un hallazgo sistémico típicamente asociado con el tipo de mordedura de araña.

Es muy importante saber valorar los daños psicológicos y traumáticos que producen estos artrópodos. También es necesario hacer campañas dentro de los centros de salud y hospitalarios, para que el médico responsable de urgencias, efectúe un estudio clínico y epidemiológico detallado, para evitar secuelas y posteriores complicaciones. (12.13)

\section{Referencias bibliográficas}

1,Geoff rey K Isbister, Hui Wen Fan, Spider Bite, Lancet 2011; 378: 2039-47 Published Online July 14, 2011.

2. D’Suze G, Corzo G, Paniagua J. Emergencia por animales ponzoñosos en las Américas. México: Instituto Bioclon; 2011.

3. OrdunaT, Lloveras S, De Roodt A,CostaV, García S, Haas A. Guía de prevención, diagnóstico, tratamiento y vigilancia epidemiológica de los envenenamientos por arañas. Buenos Aires: Ministerio de Salud de la Nación, Programa Nacional de Prevención y Control de las Intoxicaciones; 2012. 
6. Simó M, Brescovit AD. Revision and cladistic analysis of neotropical spider genus phoneutriaperty, 1833 (Aranae, ctenidae), with notes of related ctenidae. Bull Br Arachnol Soc. 2001;12(2):67-82.

7. Lise F, Mello FR. Epidemiologia do araneísmo no municipio de chapeco, santa Catarina, Brasil. SEMINA. 2007;28(2):93-8.

8. Bucaretchi F, Mello S, Vieira R, Mamoni R, Souza M, Antunes E, et al. Systemic envenomation caused by the wondering spider Phoneutria nigriventer, with quantification of circulation venom. Clinical Toxicology. 2008;46(9):885-9.

9. Tay Zavala, Jorge; GERARDO Díaz Joel; SÁNCHEZ Vega; PICADURAS POR

ALACRANES Y ARAÑAS PONZOÑOSAS DE MÉXICO, Parasitología, Facultad de Medicina, UNAM. 2 Unidad de Medicina Familiar No. 28 "Gabriel Mancera”, IMSS Enero-Febrero, 2004

10. Gomes PC, de Souza BM, Dias NB, Cesar-Tognoli LMM, Silva-Filho LC, Tormena CF, et al. Nigriventrina: a low molecular mass neuroactive compound from the venom of the spider Phoneutria nigriventer. Toxicon. 2011;57(2):266-74.

11. Diaz JH. The global epidemiology, syndromic classification, management and prevention of spider bites. Am J Trop Med Hyg. 2004;71(2):239-50.

12. Vetter Richard S, MS David L Swanson, Md Mordeduras de Arañas Reclusas, Up To Date, Revisión de la literatura actual: Aug 2017.

13. Vetter Richard S, MS David L Swanson, Abordaje del paciente con una posible picadura de araña: Panorama general, Up To Date, Revisión de la literatura actual: Aug 2017. 